УДК 519.51

\author{
I. V. Protasov, K. D. Protasova
}

\title{
LATTICES OF COARSE STRUCTURES
}

I. V. Protasov, K. D. Protasova. Lattices of coarse structures, Mat. Stud. 48 (2017), 115-123.

We consider the lattice of coarse structures on a set $X$ and study metrizable, locally finite and cellular coarse structures on $X$ from the lattice point of view.

\section{To Michael Zarichnyi on 60th birthday}

1. Introduction. Following [11], we say that a family $\mathcal{E}$ of subsets of $X \times X$ is a coarse structure on a set $X$ if

- each $\varepsilon \in \mathcal{E}$ contains the diagonal $\triangle_{X}:=\{(x, x): x \in X\}$;

- if $\varepsilon, \delta \in \mathcal{E}$ then $\varepsilon \circ \delta \in \mathcal{E}$ and $\varepsilon^{-1} \in \mathcal{E}$ where $\varepsilon \circ \delta:=\{(x, y): \exists z((x, z) \in \varepsilon,(z, y) \in \delta)\}$, $\varepsilon^{-1}:=\{(y, x):(x, y) \in \varepsilon\}$

- if $\varepsilon \in \mathcal{E}$ and $\triangle_{X} \subseteq \varepsilon^{\prime} \subseteq \varepsilon$ then $\varepsilon^{\prime} \in \mathcal{E}$.

Each $\varepsilon \in \mathcal{E}$ is called an entourage of the diagonal. We note that $\mathcal{E}$ is closed under finite unions (as $\varepsilon \cup \delta \subseteq \varepsilon \delta$ ), but $\mathcal{E}$ is not an ideal in the Boolean algebra of all subsets of $X \times X$ because $\mathcal{E}$ is not closed under formation of all subsets of its members.

A subset $\mathcal{E}^{\prime} \subseteq \mathcal{E}$ is called a base for $\mathcal{E}$ if, for every $\varepsilon \in \mathcal{E}$ there exists $\varepsilon^{\prime} \in \mathcal{E}^{\prime}$ such that $\varepsilon \subseteq \varepsilon^{\prime}$

The pair $(X, \mathcal{E})$ is called a coarse space. For $x \in X$ and $\varepsilon \in \mathcal{E}$, we denote $B(x, \varepsilon)=\{y \in$ $X:(x, y) \in \varepsilon\}$ and say that $B(x, \varepsilon)$ is a ball of radius $\varepsilon$ around $x$. We note that a coarse space can be considered as an asymptotic counterpart of a uniform topological space and could be defined in terms of balls, see [7], [9]. In this case a coarse space is called a ballean. For categorical look at the balleans and coarse structures as two faces of the same coin, see [1].

A coarse structure $\mathcal{E}$ on $X$ is called connected if, for any $x, y \in X$, there is $\varepsilon \in \mathcal{E}$ such that $y \in B(x, \varepsilon)$. A subset $Y$ of $X$ is called bounded if there exist $x \in X$ and $\varepsilon \in \mathcal{E}$ such that $Y \subseteq B(x, \varepsilon)$. A coarse structure $\mathcal{E}$ is called bounded if $X$ is bounded, otherwise $\mathcal{E}$ is called unbounded. We note that on every set $X$, there exists the unique connected bounded coarse structure $\left\{\varepsilon \subseteq X \times X: \triangle_{X} \subseteq \varepsilon\right\}$, and if $X$ is finite this structure is the unique connected coarse structure on $X$.

In what follows, we consider only connected coarse structures on infinite sets.

Given a set $X$, the family $\mathcal{L}_{X}$ of all coarse structures on $X$ is partially ordered by the inclusion, and $\mathcal{L}_{X}$ can be considered as a lattice with the operations $\wedge$ and $\vee$ :

- $\mathcal{E} \wedge \mathcal{E}^{\prime}$ is the strongest coarse structure such that $\mathcal{E} \wedge \mathcal{E}^{\prime} \subseteq \mathcal{E}, \mathcal{E} \wedge \mathcal{E}^{\prime} \subseteq \mathcal{E}^{\prime}$;

2010 Mathematics Subject Classification:06B05.

Keywords: coarse structure; ballean; lattice of coarse structures.

doi:10.15330/ms.48.2.115-123

(C) I. V. Protasov, K. D. Protasova, 2017 
- $\mathcal{E} \vee \mathcal{E}^{\prime}$ is the weakest coarse structure such that $\mathcal{E} \subseteq \mathcal{E} \vee \mathcal{E}^{\prime}, \mathcal{E}^{\prime} \subseteq \mathcal{E} \vee \mathcal{E}^{\prime}$

More explicitly, we have

$(\wedge) \quad \mathcal{E} \wedge \mathcal{E}^{\prime}=\left\{\varepsilon \cap \varepsilon^{\prime}: \varepsilon \in \mathcal{E}, \varepsilon^{\prime} \in \mathcal{E}^{\prime}\right\}$.

To clarify the operation $\vee$, we note that $\mathcal{E} \vee \mathcal{E}^{\prime}$ is the smallest coarse structure on $X$ containing $\mathcal{E}, \mathcal{E}^{\prime}$ and closed under the operations $\circ$ and ${ }^{-1}$ and taking subsets of its members containing $\triangle_{X}$. Since $(\varepsilon \circ \delta)^{-1}=\delta^{-1} \circ \varepsilon^{-1}$, the usage of the inversion is superfluous. Hence, we have

$(\vee) \mathcal{E} \vee \mathcal{E}^{\prime}$ has the base $\left\{\varepsilon_{1} \circ \ldots \circ \varepsilon_{n}: n \in \mathbb{N}, \varepsilon_{i} \in \mathcal{E} \cup \mathcal{E}^{\prime}\right\}$

The coarse structure $\mathbf{1}_{X}=\left\{\varepsilon: \triangle_{X} \subseteq \varepsilon\right\}$ is the unit of the lattice $\mathcal{L}_{\mathcal{X}}$, and the coarse structure $\mathbf{0}_{X}=\left\{\varepsilon: \triangle_{X} \subseteq \varepsilon, \varepsilon \backslash \triangle_{X}\right.$ is finite $\}$ is the null of $\mathcal{L}_{X}$.

The purpose of this note is to explore lattice properties of some basic coarse structures on a set $X$, namely, metrizable, locally finite and cellular structures. The main results are exposed in section 4 and concern the $\vee$-decomposability of coarse structure.

2. Metrizable structures. Every metric $d$ on a set $X$ defines the coarse structure $\mathcal{E}_{d}$ on $X$ with the base $\{(x, y): d(x, y) \leq n\}, n \in \omega$. A coarse structure $\mathcal{E}$ on $X$ is called metrizable if there exists a metric $d$ on $X$ such that $\mathcal{E}=\mathcal{E}_{d}$. By [9, Theorem 2.1.1], $\mathcal{E}$ is metrizable if and only if $\mathcal{E}$ has a countable base. In view of $(\wedge)$ and $(\vee)$, the family $\mathfrak{M}_{X}$ of all metrizable structures on $X$ is a sublattice of $\mathcal{L}_{X}$. Since $\mathbf{1}_{X}$ is metrizable, $\mathfrak{M}_{X}$ has the unit. If $X$ is countable then $\mathbf{O}_{X}$ is metrizable so $\mathfrak{M}_{X}$ has the null. In Proposition 1, we show that $\mathfrak{M}_{X}$ does not have null if $X$ is uncountable.

We use the following simple construction. Let $(X, d)$ and $(Y, \rho)$ be metric spaces such that $X \cap Y=\varnothing$. We take $x_{0} \in X, y_{0} \in Y, r>0$ and define a metric $\mu$ on $X \bigcup Y$ by the following rule: $\mu\left(x, x^{\prime}\right)=d\left(x, x^{\prime}\right)$ if $x, x^{\prime} \in X ; \mu\left(y, y^{\prime}\right)=\rho\left(y, y^{\prime}\right)$ if $\left(y, y^{\prime}\right) \in Y$; $\mu(x, y)=d\left(x, x_{0}\right)+r+\rho\left(y, y_{0}\right)$ if $x \in X, y \in Y$. The obtained metric space $(X \bigcup Y, \mu)$ is called the $\left(x_{0}, y_{0}, r\right)$-join of $(X, d)$ and $(Y, \rho)$.

Proposition 1. If an unbounded metric space $(X, d)$ contains an infinite bounded subset $Y$ then there exists a metric $\mu$ on $X$ such that $\mathcal{E}_{\mu} \subset \mathcal{E}_{d}$.

Proof. We pick $m \in \mathbb{N}$ such that $d\left(y, y^{\prime}\right)<m$ for all $y, y^{\prime} \in Y$ and choose an unbounded metric $\rho$ such that $\rho\left(y, y^{\prime}\right)>m$ for all $y, y^{\prime} \in Y$. Then we take $x_{0} \in X, y_{0} \in Y$, put $r=d\left(x_{0}, y_{0}\right)$ and consider the $\left(x_{0}, y_{0}, r\right)$-join $(X, \mu)$ of $(X \backslash Y, d)$ and $(Y, \rho)$. By the choice of $\mu, \mathcal{E}_{\mu} \subseteq \mathcal{E}_{d}$. Since $Y$ is unbounded in $(X, \mu)$, we have $\mathcal{E}_{\mu} \subset \mathcal{E}_{d}$.

If $X$ is uncountable, then some ball in $(X, d)$ must be infinite and, applying Proposition 1 , we get $\mathcal{E}_{\mu}$ such that $\mathcal{E}_{\mu} \subset \mathcal{E}_{d}$.

Proposition 2. For every unbounded metric space $(X, d)$, there exists an unbounded metric $\rho$ on $X$ such that $\mathcal{E}_{d} \subset \mathcal{E}_{\rho}$.

Proof. We choose a sequence $\left(a_{n}\right)_{n \in \omega}$ in $X$ such that $B_{d}\left(a_{n}, n\right) \cap B_{d}\left(a_{m}, n\right)=\varnothing$ for all $n>m$. Then we denote $Y=\left\{a_{2 n}: n \in \omega\right\}, \delta=\triangle_{X} \cup\{(x, y): x, y \in Y\}$ and put $\mathcal{H}=$ $\left\{\varepsilon_{0} \circ \ldots \circ \varepsilon_{n}: \varepsilon_{i} \in \mathcal{E}_{d} \cup\{\delta\}\right\}$. Then $\mathcal{H}$ is the base for some uniquely defined metrizable coarse structure $\mathcal{E}$ on $X$ so $\mathcal{E}=\mathcal{E}_{\rho}$ for some metric $\rho$ on $X$. We note that $\mathcal{E}_{d} \subset \mathcal{E}$ because $Y$ is bounded in $\mathcal{E}$ but $Y$ is unbounded in $\mathcal{E}_{d}$. To prove that $\mathcal{E}$ is unbounded, we choose $y_{0} \in Y$ and observe by induction on $n$ that, for each ball $B\left(y_{0}, \varepsilon_{0} \circ \ldots \circ \varepsilon_{n}\right), \varepsilon_{i} \in \mathcal{E}$, there exists $m \in \omega$ such that $B\left(y_{0}, \varepsilon_{0} \circ \ldots \circ \varepsilon_{n}\right) \subseteq B_{d}(Y, m)$ where $B_{d}(Y, m)=\cup_{y \in Y} B_{d}(y, m)$. By the choice of $\left(a_{n}\right)_{n \in \omega}, a_{k} \notin B_{d}(Y, m)$ for each odd $k>m$. 
Proposition 3. Let $d, \mu$ be unbounded metrics on a set $X$ such that $\mathcal{E}_{d} \subset \mathcal{E}_{\mu}$. Then there exists a metric $\rho$ on $X$ such that $\mathcal{E}_{d} \subset \mathcal{E}_{\rho} \subset \mathcal{E}_{\mu}$.

Proof. Since $\mathcal{E}_{d} \subset \mathcal{E}_{\mu}$, we can choose $m \in \omega$ and $a_{n}, c_{n}, n \in \omega$ such that

(1) $d\left(a_{n}, c_{n}\right)>n$

(2) $\mu\left(a_{n}, c_{n}\right)<m$.

We denote $A=\left\{a_{n}: n \in \omega\right\}, C=\left\{c_{n}: n \in \omega\right\}$ and consider two cases.

Case: $A \cup C$ is unbounded in $(X, \mu)$. We assume that $C$ is unbounded. Passing to subsequences, we may suppose that

(3) $B_{\mu}\left(c_{n}, n\right) \cap B_{\mu}\left(c_{k}, k\right)=\varnothing$ for all distinct $n, k \in \omega$.

We put $\delta=\triangle_{X} \cup\left\{\left(a_{2 n}, c_{2 n}\right),\left(c_{2 n}, a_{n}\right): n \in \omega\right\}$ and a coarse structure $\mathcal{E}$ on $X$ with the base $\mathcal{H}=\left\{\varepsilon_{0} \circ \ldots \circ \varepsilon_{n}: \varepsilon_{i} \in \mathcal{E}_{d} \cup\{\delta\}\right\}$.

Clearly, $\mathcal{E}$ is metrizable so $\mathcal{E}=\mathcal{E}_{\rho}$ for some metric $\rho$ on $X$. Since $\mathcal{E}_{d} \subseteq \mathcal{H}$, we have $\mathcal{E}_{d} \subseteq \mathcal{E}_{\rho}$. By (1), $\mathcal{E}_{d} \subset \mathcal{E}_{\rho}$.

By the choice of $\delta$ and $(2), \mathcal{E}_{\rho} \subseteq \mathcal{E}_{\mu}$. We take an arbitrary $\varepsilon_{0} \circ \ldots \circ \varepsilon_{n} \in \mathcal{H}$, delete all $\varepsilon_{i}, \varepsilon_{i}=\delta$ and get $\varepsilon_{0}^{\prime} \circ \ldots \circ \varepsilon_{s}^{\prime}$. Since $\varepsilon_{0}^{\prime} \in \mathcal{E}_{d}, \ldots, \varepsilon_{s}^{\prime} \in \mathcal{E}_{d}$, there exists $t \in \omega$ such that $B\left(x, \varepsilon_{0}^{\prime} \circ \ldots \circ \varepsilon_{s}^{\prime}\right) \subseteq B_{d}(x, t)$ for each $x \in X$. If $2 k+1>t$ then by (1), (3) and the choice of $d$, we have $a_{2 k+1} \notin B\left(c_{2 k+1}, \varepsilon_{0} \circ \ldots \circ \varepsilon_{n}\right)$. By $(2), a_{2 k+1} \in B_{\mu}\left(c_{2 k+1}, m\right)$. Hence, $\mathcal{E}_{d} \subset \mathcal{E}$.

Case: $A \cup B$ is bounded $(X, \mu)$. By (1), either $A$ or $C$ is unbounded in $(X, d)$. We assume that $A$ is unbounded in $(X, d)$. Passing to subsequences, we may suppose that $B_{d}\left(a_{n}, n\right) \cap$ $B_{d}\left(a_{k}, n\right)=\varnothing$ for all $n>k$. Since $A$ is bounded in $(X, \mu)$, we can apply arguments from the proof of Proposition 2.

3. Locally finite structures. A coarse structure $\mathcal{E}$ on a set $X$ is called locally finite if each ball $B(x, \varepsilon), x \in X, \varepsilon \in \mathcal{E}$ is finite. If for every $\varepsilon \in \mathcal{E}$ there exists $m \in \omega$ such that, for each $x \in X,|B(x, \varepsilon)|<m$ then $\mathcal{E}$ is called uniformly locally finite. The uniformly locally finite coarse structures play the central part in Asymptotic Topology, see [2], [6].

In view $(\wedge)$ and $(\wedge)$, the families of locally finite and uniformly locally finite coarse structures on $X$ are sublattices of $\mathcal{L}_{X}$ with the null $\mathbf{0}_{X}$. To find units in these structures, we use some specializations of the following general construction.

Let $G$ be a group with the identity $e$ and let $X$ be a $G$-space with the action $G \times X \longrightarrow X$, $(g, x) \longmapsto g x$. An ideal $\mathcal{I}$ in the Boolean algebra $\mathcal{P}_{G}$ of all subsets of $X$ is called a group ideal if $\mathcal{I}$ contains the ideal $\mathfrak{F}_{G}$ of all finite subsets and $A B^{-1} \in \mathcal{I}$ for any $A, B \in \mathcal{I}$. For group ideals, see [8] and [9, Chapter 6].

We assume that $G$ acts on $X$ transitively (for any $x, y \in X$, there is $g \in G$ such that $g x=y)$ and, given a group ideal $\mathcal{I}$ in $\mathcal{P}_{G}$, we consider the coarse structure $\mathcal{E}, \mathcal{E}=\mathcal{E}(G, \mathcal{I}, X)$ on $X$ with the base $\left\{\varepsilon_{A}: A \in \mathcal{I}, c \in A\right\}, \varepsilon_{A}=\{(x, g x): x \in X, g \in A\}$. Then $B\left(x, \varepsilon_{A}\right)=A x$, $A x=\{g x: g \in A\}$.

By [5, Theorem 1], for every coarse structure $\mathcal{E}$ on $X$ there exists a group $G$ of permutations of $X$ and a group ideal $\mathcal{I}$ in $\mathcal{P}_{G}$ such that $\mathcal{E}=\mathcal{E}(G, \mathcal{I}, X)$.

Every coarse structure $\mathcal{E}\left(G, \mathfrak{F}_{G}, X\right)$ is uniformly locally finite. If $X=G$ and $G$ acts of $X$ by the left shifts then $\mathcal{E}\left(G, \mathfrak{F}_{G}, G\right)$ is called the finitary coarse structure on $G$. The finitary coarse structures on finitely generated groups take an impotant part of Geometrical Group Theory, see [4, Chapter 4].

By [6, Theorem 1], for every uniformly locally finite coarse structure $\mathcal{E}$ on $X$, there exists a group $G$ of permutations of $X$ such that $\mathcal{E}=\mathcal{E}\left(G, \mathfrak{F}_{G}, X\right)$. Applying this theorem, we get 
Proposition 4. Let $X$ be a set and let $S_{X}$ denotes the group of all permutations of $X$. Then the coarse structure $\mathcal{E}\left(S_{X}, \mathfrak{F}_{S_{X}}, X\right)$ is the unit in the lattice of all uniformly locally finite coarse structures on $X$.

We endow $S_{X}$ with the topology of pointwise convergence, the subsets $\left\{g \in S_{X}: g x=y\right\}$, $x, y \in X$ form a sub-base of this topology. We note that a subset $A$ of $S_{X}$ is precompact if and only if the set $A x$ is finite for each $x \in X$. We denote by $\mathfrak{C}_{S_{X}}$ the ideal of all precompact subsets of $S_{X}$.

Proposition 5. The coarse structure $\mathcal{E}\left(S_{X}, \mathfrak{C}_{S_{X}}, X\right)$ is the unit in the lattice of all locally finite coarse structures on $X$.

Proof. Let $\mathcal{E}^{\prime}$ be a locally finite and let $\varepsilon \in \mathcal{E}^{\prime}$. For each $(x, y) \in \mathcal{E}$, we take a permutation $f_{(x, y)}$ such that $f(x)=y, f(y)=x$ and $f(z)=z$ for all $z \in X \backslash\{x, y\}$. We put $A=$ $\left\{f_{x, y}:(x, y) \in \varepsilon\right\}$. Since $\mathcal{E}$ is locally finite, we have $A \in \mathfrak{C}_{S_{X}}$. By the choice of $A, \varepsilon=\varepsilon_{A}$ so $\varepsilon \in \mathcal{E}\left(S_{X}, \mathfrak{C}_{S_{X}}, X\right)$.

We recall that two metric space $(X, d),(Y, \rho)$ are isometric if there is a bijection $f: X \rightarrow$ $Y$ such that $d(x, y)=\rho(f(x), f(y))$ for all $x, y \in Y$. If a metrizable coarse structure on $X$ is locally finite then $X$ must be countable.

Proposition 6. Let $d, \rho$ be unbounded metrics on $X$ such that $(X, d),(X, \rho)$ are locally finite. Then there exists a metric $\mu$ on $X$ such that $\mathcal{E}_{d} \wedge \mathcal{E}_{\mu}=\mathbf{0}_{X}$ and $(X, \rho),(X, \mu)$ are isometric.

Proof. Assume that we have defined $\mu$ so that, for every $n \in \omega$ there exists a finite subset $F$ of $X$ such that if $x \in X \backslash F, y \in X, y \neq x$ and $d(x, y)<n$ then $\mu(x, y)>n$. Then $B_{d}(x, n) \cap B_{\mu}(x, n)=\{x\}$ and $\mathcal{E}_{d} \wedge \mathcal{E}_{\mu}=\mathbf{0}_{X}$. The problem is to find $\mu$ so that $(X, \rho),(X, \mu)$ are isometric and $\mu$ satisfies the above conditions. We construct inductively some bijection $f: X \rightarrow X$ and get $\mu$ as $\mu(x, y)=\rho(f(x), f(y))$.

We choose a sequence $\left(a_{n}\right)_{n \in \omega}$ in $X$ such that $B_{d}\left(a_{n}, n\right) \cap B_{d}\left(a_{m}, m\right)=\varnothing$ for all distinct $n, m \in \omega$. Then we take a numeration $X=\left\{x_{n}: n \in \omega\right\}$ satisfying the following conditions. If $x_{i} \in B_{d}\left(a_{n}, n\right), x_{j} \in B_{d}\left(a_{m}, m\right)$ and $n<m$, then $i<j$. For every $n \in \omega, B_{d}\left(a_{n}, n\right)=$ $\left\{x_{k_{n}}, x_{k_{n}+1}, \ldots, x_{s_{n}}\right\}, x_{k_{n}}=a_{n}$. We choose a sequence $\left(b_{n}\right)_{n \in \omega}$ in $X$ such that $B_{\rho}\left(b_{n}, n\right) \cap$ $B_{\rho}\left(b_{m}, m\right)=\varnothing$ for all distinct $n, m \in \omega$.

We put $f\left(x_{0}\right)=b_{0}$ and suppose that we have defined $f\left(x_{0}\right), \ldots, f\left(x_{k}\right)$. If $x_{k+1} \in X \backslash$ $\left\{a_{n}: n \in \omega\right\}$ then we put $f\left(x_{k+1}\right)=b_{k+1}$.

If $x_{k}=a_{n}$ and $X \backslash\left(\left\{f\left(x_{0}\right), \ldots, f\left(x_{k}\right)\right\} \cup \bigcup_{i>k} B_{\rho}\left(b_{i}, i\right)\right)=\varnothing$, then we put $f\left(x_{k+1}\right)=b_{k+1}$. Otherwise, we take the minimal $j$ such that $x_{j} \in X \backslash\left(\left\{f\left(x_{0}\right), \ldots, f\left(x_{k}\right)\right\} \cup \bigcup_{i>k} B_{\rho}\left(b_{i}, i\right)\right)$ and put $f\left(x_{k+1}\right)=x_{j}$.

After $\omega$ steps, we get the injective mapping $f: X \rightarrow X$. To see that $f$ is surjective, it suffices note that $\cap_{k \in \omega} \bigcup_{i>k} B_{\rho}\left(b_{i}, i\right)=\varnothing$.

At last, if $n \in \omega, k>n, y \neq x_{k}$ and $d\left(x_{k}, y\right)<n, y \neq x_{k}$ then $f(y) \notin B_{\rho}\left(f\left(x_{k}\right), n\right)$ so $\rho\left(f\left(x_{k}\right), f(y)\right)>n$.

Proposition 7. Let $(X, d)$ be a locally finite metric space. Then there exists a metric $d^{\prime}$ on $X$ such that $\left(X, d^{\prime}\right)$ is locally finite and $E_{d} \subset E_{d^{\prime}}$.

Proof. If $E_{d}=\mathbf{0}_{X}$ then the statement is evident. We suppose that $E_{d} \neq \mathbf{0}_{X}$, put $\rho=d$ and applying Proposition 6 get the locally finite metric $\mu$ on $X$ such that $\mathcal{E}_{\mu} \wedge \mathcal{E}_{d}=\mathbf{0}_{X}$ and $(X, \mu)$ is isometric to $(X, d)$. Since $\mathcal{E}_{d} \neq \mathbf{0}_{X}$, we have $\mathcal{E}_{d} \subset \mathcal{E}_{d} \vee \mathcal{E}_{\mu}$ and $\mathcal{E}_{d} \vee \mathcal{E}_{\mu}=\mathcal{E}_{d^{\prime}}$ for some locally finite metric $d^{\prime}$ on $X$. 
Proposition 8. Let $(X, d),(X, \mu)$ be locally finite metric spaces such that $\mathcal{E}_{d} \subset \mathcal{E}_{\mu}$. Then there exists a locally finite metric $\rho$ on $X$ such that $\mathcal{E}_{d} \subset \mathcal{E}_{\rho} \subset \mathcal{E}_{\mu}$.

Proof. We apply Proposition 3 and note that $\rho$ is locally finite because $\mathcal{E}_{\rho} \subset \mathcal{E}_{\mu}$.

By Proposition 7, the unit in the lattice of all locally finite coarse structures on $X$ (see Proposition 5) is not metrizable. It is easy to see that Proposition 7 remains true with uniformly locally finite metrics in place of locally finite metrics, so the unit in the lattice of all uniformly locally finite metric spaces (see Proposition 4) is not metrizable.

4. Cellular structures. Every equivalence $\varepsilon$ on a set $X$ partitions $X$ into equivalence classes. On the other hand, every partition $\mathcal{P}$ of $X$ defines the equivalence $\varepsilon$ by the rule: $(x, y) \in \varepsilon$ if and only if $x, y$ are in some cell of the partition $\mathcal{P}$.

A coarse structure $\mathcal{E}$ on a set $X$ is called cellular if $\mathcal{E}$ has a base consisting of the equivalence relations.

Given an equivalence $\varepsilon$ on $X$, we denote by $\langle\varepsilon\rangle$ the smallest coarse structure on $X$ such that $\varepsilon \in\langle\varepsilon\rangle$ and say that $\langle\varepsilon\rangle$ is generated by $\varepsilon$. To show that $\langle\varepsilon\rangle$ is cellular, we take the partition $\mathcal{P}$ into $\varepsilon$-classes and, for each finite subset $\mathfrak{F}$ of $\mathcal{P}$, denote by $\mathcal{P}_{\mathfrak{F}}$ the partition $\{\cup \mathfrak{F}\} \cup(\mathcal{P} \backslash \mathfrak{F})$ of $X$. We denote by $\varepsilon_{\mathfrak{F}}$ the equivalence defined by the partition $\mathcal{P}_{\mathfrak{F}}$ and observe that $\left\{\varepsilon_{\mathfrak{F}}: \mathfrak{F}\right.$ is a finite subset of $\left.\mathcal{P}\right\}$ is a base for $\langle\varepsilon\rangle$.

By [9, Theorem 3.11], a metrizable coarse structure $\mathcal{E}$ on $X$ is cellular if and only if $\mathcal{E}=\mathcal{E}_{d}$ for some ultrametric $d$ on $X$. By [9, Theorem 3.1.3], a coarse structure $\mathcal{E}$ on $X$ is cellular if and only if $\operatorname{asdim}(X, \mathcal{E})=0$. For definition of the asymptotic dimension see $[9$, p.45] or [11, p. 129].

If $\mathcal{E}_{1}, \mathcal{E}_{2}$ are cellular coarse structures on $X$, then $\mathcal{E}_{1} \wedge \mathcal{E}_{2}$ is cellular, but the set of all cellular coarse structure on $X$ is not closed under the operation $\vee$. Moreover, at the moment we may even conjecture that every coarse structure $\mathcal{E}$ on $X$ can be represented as $\mathcal{E}=\mathcal{E}_{1} \vee \mathcal{E}_{2}$ for some cellular coarse structures on $X$. After two examples, we put this insolent conjecture in restricted form of open questions.

We say that a coarse structure $\mathcal{E}$ on $X$ is $\vee$-decomposable if there exist two cellular coarse structures $\mathcal{E}_{0}, \mathcal{E}_{1}$ on $X$ such that $\mathcal{E}=\mathcal{E}_{0} \vee \mathcal{E}_{1}$.

Example 1. For $n \in \mathbb{N}$, we endow $\mathbb{Z}^{n}$ with the metric $d(x, y)=\left|x_{1}-y_{1}\right|+\ldots+\left|x_{n}-y_{n}\right|$, put $\mathcal{E}=\mathcal{E}_{d}$ and show that $\mathcal{E}$ is $\vee$-decomposable.

We put $K=\left\{x \in \mathbb{Z}^{n}: x_{i} \in\{0,1\}\right\}$ and consider the partitions $\mathcal{P}_{0}=\left\{K+a: a \in 2 \mathbb{Z}^{n}\right\}$, $\mathcal{P}_{1}=\left\{(1,1, \ldots, 1)+P: P \in \mathcal{P}_{0}\right\}$. Let $\varepsilon_{0}, \varepsilon_{1}$ be the equivalences defined by $\mathcal{P}_{0}, \mathcal{P}_{1}$, and $\mathcal{E}_{0}$, $\mathcal{E}_{1}$ be the coarse structures generated by $\varepsilon_{0}$, $\varepsilon_{1}$. Clearly, $\mathcal{E}_{0} \subset \mathcal{E}, \mathcal{E}_{1} \subset \mathcal{E}$ so $\mathcal{E}_{0} \vee \mathcal{E}_{1} \subseteq \mathcal{E}$. If $x, y \in \mathbb{Z}^{n}$ and $d(x, y)=1$ then there exists $P \in \mathcal{P}_{0} \cup \mathcal{P}_{1}$ such that $\{x, y\} \subset P$. It follows that $\mathcal{E} \subseteq \mathcal{E}_{0} \vee \mathcal{E}_{1}$

Let $\Gamma$ be a connected graph with the set of vertices $\mathcal{V}$. We endow $\mathcal{V}$ with the path metric $d$ and denote by $\mathcal{E}_{\Gamma}$ the coarse structure $\mathcal{E}_{d}$ on $\mathcal{V}$. Given a coarse structure $\mathcal{E}$ on a set $X$, how one can detect if there exists a graph $\Gamma$ with the set of vertices $X$ such that $\mathcal{E}=\mathcal{E}_{\Gamma}$ ? The answer to this question gives Theorem 5.1.1 from [9].

Example 2. Let $\mathcal{T}$ be a tree. Then the coarse structure $\mathcal{E}_{\mathcal{T}}$ is $\vee$-decomposable, see Proposition 9 for more general result.

Question 1. Is every uniformly locally finite coarse structure $\vee$-decomposable?

Question 2. Is the coarse structure $\mathcal{E}_{\Gamma} \vee$-decomposable for every connected graph $\Gamma$ ? 
We note that the finitary coarse structures of finitely generated groups lie in the intersection of Questions 1 and 2.

Let $\left(\Gamma_{n}\right)_{n \in \omega}$ be a sequence of finite connected graphs with pairwise disjoint sets of vertices $\left\{\mathcal{V}_{n}: n \in \omega\right\}$. For each $n \in \omega$, we pick $v_{n} \in \mathcal{V}_{n}$ and join $\Gamma_{n}$ and $\Gamma_{n+1}$ by the edge $\left\{v_{n}, v_{n+1}\right\}$. We believe that the resulting graph $\Gamma$ is a good candidate for counterexamples to both questions if $\left(\Gamma_{n}\right)_{n \in \omega}$ is a family of expanders. What is an expander, see Wikipedia.

Proposition 9. Let $\Gamma$ be a graph with the set of vertices $\mathcal{V}, v_{0} \in \mathcal{V}, S\left(v_{0}, n\right)=\{v \in$ $\left.\mathcal{V}: d\left(v_{0}, v\right)=n\right\}$. We denote by $\Gamma_{n}$ the subgraph of $\Gamma$ with the set of vertices $S\left(v_{0}, n\right) \cup$ $S\left(v_{0}, n+1\right)$ and assume that there exists $k \in \mathbb{N}$ such that, for every $n \in \omega$, each connected component of $\Gamma_{n}$ is contained in some ball of radius $k$ in $\Gamma$.

Then the coarse structure $\mathcal{E}_{\Gamma}$ is $\vee$-decomposable.

Proof. We denote by $\mathcal{P}_{0}$ the partition of $\mathcal{V}$ into connected components of $\Gamma_{2 n}, n \in \omega$, and by $\mathcal{P}_{1}$ the partition of $\mathcal{V} \backslash\left\{v_{0}\right\}$ into connected components of $\Gamma_{2 n+1}, n \in \omega$. Then we take the equivalences $\varepsilon_{0}, \varepsilon_{1}$ on $\mathcal{V}$ defined by the partitions $\mathcal{P}_{0}$ and $\mathcal{P}_{1},\left\{v_{0}\right\}$, and denote by $\mathcal{E}_{0}, \mathcal{E}_{1}$ the coarse structures on $\mathcal{V}$ generated by $\mathcal{E}_{0}$ and $\mathcal{E}_{1}$. By the assumption, $\mathcal{E}_{0} \subseteq \mathcal{E}_{\Gamma}, \mathcal{E}_{1} \subseteq \mathcal{E}_{\Gamma}$ so $\mathcal{E}_{0} \vee \mathcal{E}_{1} \subseteq \mathcal{E}_{1}$. Since every edge of $\Gamma$ is an edge of some connected component of $\Gamma_{m}$, we have $\mathcal{E}_{\Gamma} \subseteq \mathcal{E}_{0} \vee \mathcal{E}_{1}$

We note that beside trees, Proposition 9 demonstrates $\vee$-decomposability of $\mathcal{E}_{\Gamma}$ provided that there exists $k \in \mathbb{N}$ such that $\left|S\left(v_{0}, n\right)\right| \leq k$ for each $n \in \omega$.

Proposition 10. Assume that there exists a partition $\mathcal{P}$ of the set $\mathcal{V}$ of vertices of a graph $\Gamma$ and $r \in \mathbb{N}$ such that, for every $P \in \mathcal{P}$ the following conditions hold:

(i) $\quad P \subseteq B(v, r)$ for some $v \in \mathcal{V}$;

(ii) $\quad\left|\left\{P^{\prime} \in \mathcal{P}: P \neq P^{\prime}, \quad P^{\prime} \cap B(P, 1) \neq \varnothing\right\}\right| \leq|P|$.

Then the coarse structure $\mathcal{E}_{\Gamma}$ is $\vee$-decomposable.

Proof. We denote $\mathcal{N}(P)=\left\{P^{\prime} \in \mathcal{P}: P \neq P^{\prime}, P^{\prime} \cap B(P, 1) \neq \varnothing\right\}$ and suppose that we have chosen a family $\mathfrak{F}$ of two-element pairwise disjoint subsets of $\mathcal{V}$ such that

(*) for any $P \in \mathcal{P}$ and $Q \in \mathcal{N}(P)$, there exists $F \in \mathfrak{F}$ such that $|P \cap F|=|Q \cap F|=1$, and for any $F \in \mathfrak{F}$, there exist $P \in \mathcal{P}, Q \in \mathcal{N}(P)$ such that $|P \cap F|=|Q \cap F|=1$.

We take the equivalences $\varepsilon, \gamma$ on $\mathcal{V}$ defined by the partitions $\mathcal{P}$ and $\mathfrak{F},\{v\}, v \in \mathcal{V} \backslash \cup \mathfrak{F}$, and denote by $\mathcal{E}_{0}$ and $\mathcal{E}_{1}$ the coarse structures on $\mathcal{V}$ generated by $\varepsilon$ and $\gamma$. We observe that $\mathcal{E}_{0}, \mathcal{E}_{1}$ are cellular and, by $(i),(*), \mathcal{E}_{0} \subseteq \mathcal{E}_{\Gamma}, \mathcal{E}_{1} \subseteq \mathcal{E}_{\Gamma}$ so $\mathcal{E}_{0} \vee \mathcal{E}_{1} \subseteq \mathcal{E}_{\Gamma}$.

On the other hand, let $\{x, y\}$ be an edge of $\Gamma$. If $\{x, y\} \subseteq P$ for some $P \in \mathcal{P}$, then $(x, y) \in \varepsilon$. Otherwise, there exist $P \in \mathcal{P}$ and $Q \in \mathcal{N}(P)$ such that $x \in P, y \in Q$ and, by (*), $(x, y) \in \varepsilon \circ \gamma \circ \varepsilon$. Hence, $\mathcal{E}_{\Gamma} \subseteq \mathcal{E}_{0} \vee \mathcal{E}_{1}$.

To define $\mathfrak{F}$, we enumerate $\mathcal{P}=\left\{P_{\alpha}: \alpha<\kappa\right\}$ and choose an injective mapping $f_{\alpha}: \mathcal{N}\left(P_{\alpha}\right) \rightarrow P_{\alpha}$. We denote $X_{\alpha}=\left\{\beta<\alpha: P_{\beta} \in \mathcal{N}\left(P_{\alpha}\right)\right\}$ and put $\mathfrak{F}_{\alpha}=\left\{\left\{f_{\beta}\left(P_{\alpha}\right), f_{\alpha}\left(P_{\beta}\right)\right\}:\right.$ $\left.\beta \in X_{\alpha}\right\}$. Then the desired $\mathfrak{F}$ is $\bigcup_{\alpha<\kappa} \mathfrak{F}_{\alpha}$.

Proposition 11. Let $\Gamma$ be a graph with the set of vertices $\mathcal{V}$. Assume that there exists $r \in \mathbb{N}$ such that $|B(v, r)|=|\mathcal{V}|$ for each $v \in \mathcal{V}$. Then the coarse structure $\mathcal{E}_{\Gamma}$ is $\vee$-decomposable.

Proof. We use the Zorn's lemma to choose a subset $X \subset \mathcal{V}$ such that $B(x, r) \cap B\left(x^{\prime}, r\right)=\varnothing$ for all distinct $x, x^{\prime} \in X$ and, for every $v \in \mathcal{V}$, there exists $x \in X$ such that $B(v, r) \cap B(x, r) \neq \varnothing$. Then we take an arbitrary partition $\mathcal{P}$ of $\mathcal{V}$ such that if $x \in X$ and $x \in P, P \in \mathcal{P}$ then $P \subseteq B(x, 2 r)$. Apply Proposition 10 . 
We recall that a metric space $(X, d)$ is geodesic if, for any $x, y \in X$, there is an isometric embedding $f:[0, d(x, y)] \longrightarrow X$ such that $f(0)=x, f(d(x, y))=y$.

Proposition 12. Let $(X, d)$ be a geodesic metric space such that $\left|B_{d}(x, 1)\right|=|X|$ for each $x \in X$. Then the coarse structure $\mathcal{E}_{d}$ is $\vee$-decomposable.

Proof. We consider a graph $\Gamma$ with the set of vertices $X$ and the set of edges $\{\{x, y\}: 0<$ $d(x, y) \leq 1\}$. Then the coarse structures $\mathcal{E}_{d}$ and $\mathcal{E}_{\Gamma}$ coincide. Apply Proposition 11.

In all above propositions, the coarse structure $\mathcal{E}_{0}, \mathcal{E}_{1}$ witnessing the decomposition $\mathcal{E}=$ $\mathcal{E}_{0} \vee \mathcal{E}_{1}$ are generated by some equivalences $\varepsilon_{0}, \varepsilon_{1}$. If the metric space $(X, d)$ in Proposition 12 is separable, then $\mathcal{E}_{0}, \mathcal{E}_{1}$ can be chosen to be metrizable.

Let $(X, \mathcal{E})$ be a coarse space. Each non-empty subset $Y$ of $X$ has the natural coarse structure $\left.\mathcal{E}\right|_{Y}=\{\varepsilon \cap(Y \times Y): \varepsilon \in \mathcal{E}\}$ which is called a substructure of $\mathcal{E}$.

A subset $Y$ of $X$ is called large in $(X, \mathcal{E})$ if there exists $\varepsilon \in \mathcal{E}$ such that $X=B(Y, \mathcal{E})$.

Proposition 13. Let $\mathcal{E}$ be a coarse structure on a set $X$ and let $Y$ be a large subset of $X$. If $\left.\mathcal{E}\right|_{Y}$ is decomposable then $\mathcal{E}$ is $\vee$-decomposable.

Proof. We take $\varepsilon_{0} \in \mathcal{E}$ such that $\varepsilon_{0}=\varepsilon_{0}^{-1}, X=B\left(Y, \varepsilon_{0}\right)$ and choose a mapping $f: X \rightarrow Y$ such that $(x, f(x)) \in \varepsilon_{0}$ and $f(y)=y$ for every $y \in Y$. For each $\gamma \subseteq Y \times Y$, we put $f^{-1}(\gamma)=$ $\left\{\left(x, x^{\prime}\right) \in X \times X:\left(f(x), f\left(x^{\prime}\right) \in \gamma\right)\right\}$ and note that

$(*) \mathcal{U}$ is a base of $\left.\mathcal{E}\right|_{Y}$ if and only if $f^{-1}(\mathcal{U})=\left\{f^{-1}(\gamma): \gamma \in \mathcal{U}\right\}$ is a base of $\mathcal{E}$.

We assume that $\left.\mathcal{E}\right|_{Y}=\mathcal{E}_{0}^{\prime} \vee \mathcal{E}_{1}^{\prime}$ with cellular $\mathcal{E}_{0}^{\prime}, \mathcal{E}_{1}^{\prime}$ and put $\mathcal{E}_{0}=f^{-1}\left(\mathcal{E}_{0}^{\prime}\right), \mathcal{E}_{1}=f^{-1}\left(\mathcal{E}_{1}^{\prime}\right)$ and note that $\mathcal{E}_{0}, \mathcal{E}_{1}$ are cellular. If $\gamma, \gamma^{\prime} \in Y \times Y$ then $f^{-1}\left(\gamma \circ \gamma^{\prime}\right)=f^{-1}(\gamma) \circ f^{-1}\left(\gamma^{\prime}\right)$ so $f^{-1}\left(\mathcal{E}_{0}^{\prime} \vee \mathcal{E}_{1}^{\prime}\right)=\mathcal{E}_{0} \vee \mathcal{E}_{1}$ and, applying $(*)$, we get $\mathcal{E}=\mathcal{E}_{0} \vee \mathcal{E}_{1}$.

Question 3. Is every substructure of $\vee$-decomposable coarse structure $\vee$-decomposable?

In view of Proposition 12, in order to answer Question 2 in affirmative, it suffices to get the positive answer to Question 3 for substructures $\left.\mathcal{E}\right|_{Y}$ where $Y$ is a large subset of $X$. Indeed, replacing each edge $\{x, y\}$ of $\Gamma$ by the isometric copy of the unit interval, we get the geodesic metric space $(X, d)$ in which the set $\mathcal{V}$ of vertices of $\Gamma$ is large.

Each ideal $\mathcal{I}$ in the Boolean algebra $\mathcal{P}_{X}$ containing $\mathfrak{F}_{X}$ defines a cellular coarse structure $\mathcal{E}_{\mathcal{I}}$ with the base $\left\{\varepsilon_{A}: A \in \mathcal{I}\right\}$ where $\varepsilon_{A}$ is the partition $\{A\} \cup\{\{x\}: x \in X \backslash A\}$. Thus, $B\left(x, \varepsilon_{A}\right)=A$ if $x \in A$ and $B\left(x, \varepsilon_{A}\right)=\{x\}$ if $x \in X \backslash A$. If $A$ is an arbitrary subset of $X$ then the set $\mathcal{I}_{A}=\left\{A \cup F: F \in \mathfrak{F}_{X}\right\}$ is the smallest ideal such that $A \in \mathcal{I}_{A}$ and $\mathfrak{F}_{X} \subseteq \mathcal{I}_{A}$.

Given an arbitrary coarse structure $\mathcal{E}$ on $X, \mathcal{E} \neq \mathbf{1}_{X}$, the family $\mathcal{I}$ of all bounded subset of $X$ is an ideal in $\mathcal{P}_{X}$. We say that $\mathcal{E}_{\mathcal{I}}$ is the companion of $\mathcal{E}$.

An ideal $\mathcal{I}$ in $\mathcal{P}_{X}$ is maximal if and only if $\{X \backslash A: A \in \mathcal{I}\}$ is a free ultrafilters, so there are $2^{2^{|X|}}$ coarse structures on $X$ defined by the maximal ideals, in particular, $\left|\mathcal{L}_{X}\right|=2^{2^{|X|}}$.

Proposition 14. Let $\mathcal{E}$ be a cellular coarse structure on $X$ such that $\mathcal{E} \neq \mathbf{0}_{X}$ and $\mathcal{E} \neq \mathbf{1}_{X}$. Then there exists a cellular coarse structure $\mathcal{E}^{\prime}$ on $X$ such that $\mathcal{E} \vee \mathcal{E}^{\prime}=\mathbf{1}_{X}$ and $\mathcal{E}^{\prime} \neq \mathbf{1}_{X}$.

Proof. By the assumption, there exists an equivalence $\varepsilon \in \mathcal{E}$ such that either some $\varepsilon$-class $P$ is infinite and $X \backslash P$ is infinite, or there are infinitely many $\varepsilon$-classes containing at least two elements.

In both cases, we choose a subset $A$ of $X$ such that $|A \cap B|=1$ for each $\varepsilon$-class $B$, put $\mathcal{E}^{\prime}=\mathcal{E}_{\mathcal{I}_{A}}$ and observe that $\mathcal{E} \vee \mathcal{E}^{\prime}=\mathbf{1}_{X}, \mathcal{E}^{\prime} \neq \mathbf{1}_{X}$. 
Proposition 15. Let $\mathcal{I}$ be a maximal ideal in $\mathcal{P}_{X}$ and $\mathcal{E}=\mathcal{E}_{\mathcal{I}}$. If $\mathcal{E}^{\prime}$ is a coarse structure on $X$ such that $\mathcal{E} \wedge \mathcal{E}^{\prime}=\mathbf{0}_{X}$ then $\mathcal{E}^{\prime}=\mathbf{0}_{X}$.

Proof. We assume that some infinite subset of $X$ is bounded in $\vee$. Since the set $\varphi=$ $\{X \backslash A: A \in \mathcal{I}\}$ is a free ultrafilter, there is an infinite subset $A$ of $X$ bounded in $\mathcal{E}^{\prime}$ such that $A \in \mathcal{I}$. We take $\gamma \in \mathcal{E}^{\prime}$ such that $A \subseteq B(x, \gamma)$ for each $x \in A$. For every finite subset $K$ of $X$ and $x \in A \backslash K$, the set $A \cap B(x, \gamma)$ is infinite. Hence, $\mathcal{E} \wedge \mathcal{E}^{\prime} \neq \mathbf{0}_{X}$. Thus, every bounded in $\mathcal{E}^{\prime}$ subset of $X$ is finite.

Given an arbitrary $\gamma \in \mathcal{E}^{\prime}$, we denote $C=\{x \in X:|B(x, \alpha)|>1\}$ If $C$ is finite then $\mathcal{E}^{\prime}=\mathbf{0}_{X}$. Otherwise $C$ is unbounded. Since $\varphi$ is a free ultrafilter, there exists an infinite subset $C^{\prime}$ of $C$ such that $B\left(C^{\prime}, \gamma\right) \in \mathcal{I}$. By above paragraph, $\mathcal{E} \wedge \mathcal{E}^{\prime} \neq \mathbf{0}_{X}$.

Let $\mathcal{E}$ be a coarse structure on $X$. We say that a coarse structure $\mathcal{E}^{\prime}$ is a complement to $\mathcal{E}$ if $\mathcal{E} \wedge \mathcal{E}^{\prime}=\mathbf{0}_{X}, \mathcal{E} \wedge \mathcal{E}^{\prime}=\mathbf{1}_{X}$ If $\mathcal{E}$ has a complement, we say that $\mathcal{E}$ is complementable. Clearly, $\mathbf{0}_{X}$ and $\mathbf{1}_{X}$ are complementable.

Example 3. We take an infinite subset $A$ such that the set $B=X \backslash A$ is infinite, and denote $\mathcal{I}_{A}=\left\{A \cup F: F \in \mathfrak{F}_{X}\right\}, \mathcal{I}_{B}=\left\{B \cup F: F \in \mathfrak{F}_{X}\right\}$. Then $\mathcal{E}_{\mathcal{I}_{A}} \wedge \mathcal{E}_{\mathcal{I}_{B}}=\mathbf{0}_{X}, \mathcal{E}_{\mathcal{I}_{A}} \vee \mathcal{E}_{\mathcal{I}_{B}}=\mathbf{1}_{X}$.

Question 4. How one can detect whether a coarse structure on $X$ is complementable?

5. Comments. 1. By Zorn's lemma, every unbounded coarse structure on a set $X$ is contained is some maximal unbounded coarse structure. For criterion of maximality and some properties of maximal coarse structures, see [9, Chapter 10]. We mention only two facts. If $\mathcal{I}$ is a maximal ideal on $X$, then $\mathcal{E}_{\mathcal{I}}$ is maximal. If $X$ is countable, then the unit in the lattice of all uniformly finite coarse structure on $X$ (see Proposition 4 ) is maximal.

2. We recall that an element $a$ of a lattice $\mathcal{L}$ with $\mathbf{0}$ and $\mathbf{1}$ is an atom (a coatom) if $a \neq \mathbf{0}$ $(a \neq \mathbf{1})$ and $b<a(b>a)$ implies $b=\mathbf{0}(b=\mathbf{1})$.

For every set $X$, the lattice $\mathcal{L}_{X}$ has $2^{2^{|X|}}$ coatoms, see above paragraph. We show that $\mathcal{L}_{X}$ has no atoms. Assume the contrary and let $\mathcal{E}$ be an atom in $\mathcal{L}_{X}$.

We suppose that the ideal $\mathcal{I}$ of all bounded subsets of $X$ has an infinite member $A$, partition $A$ into infinite subsets $B, C$ and denote by $\mathcal{I}_{A}$ the smallest ideal in $\mathcal{P}_{X}$ containing $A$ and $\mathfrak{F}_{X}$. Then $\mathcal{E}_{\mathcal{I}_{A}} \subset \mathcal{E}_{\mathcal{I}} \subseteq \mathcal{E}, \mathcal{E}_{\mathcal{I}_{A}} \neq \mathbf{0}_{X}$. It follows that $\mathcal{I}=\mathfrak{F}_{X}$.

Since $\mathcal{E} \neq \mathbf{0}_{X}$, we can choose $\varepsilon \in \mathcal{E}$ and a sequence $\left(a_{n}\right)_{n \in \omega}$ in $X$ such that $\left|B\left(a_{n}, \varepsilon\right)\right|>1$, $B\left(a_{n}, \varepsilon\right) \cap B\left(a_{m}, \varepsilon\right)=\varnothing$ for all distinct $n, m$. For each $n \in \omega$, we take $b_{2 n} \in B\left(a_{2 n}, \varepsilon\right), b_{2 n} \neq$ $a_{2 n}$ and denote $\gamma=\left\{\left(a_{2 n}, b_{2 n}\right),\left(b_{2 n}, a_{2 n}\right): n \in \omega\right\} \cup \triangle_{X}$. We denote by $\mathcal{E}^{\prime}$ the smallest coarse structure on $X$ containing $\gamma$ : By the choice of $\gamma$ and above paragraph $\left(\mathcal{I}=\mathfrak{F}_{X}\right), \mathbf{0}_{X} \subset \mathcal{E}^{\prime}$ and, for every $\delta \in \mathcal{E}^{\prime}$, there is $m \in \omega$ such that $\left|B\left(a_{2 m+1}, \delta\right)\right|=1$. Hence, $\boldsymbol{0}_{X} \subset \mathcal{E}^{\prime} \subset \mathcal{E}$ and we run in a contradiction. If $\mathcal{E}$ is a metrizable coarse structure on $X$ and $\mathcal{E} \neq \mathbf{0}_{X}$ then Proposition 1 and these arguments give us metrizable $\mathcal{E}^{\prime}$ such that $\mathbf{0}_{X} \subset \mathcal{E}^{\prime} \subset \mathcal{E}$.

3. For a group $G$, the family $L I_{G}$ of all group ideals in $\mathcal{P}_{G}$ has the natural lattice structure. The lattices $L I_{G}$ were introduced and studied in [3], see also [9, Chapter 6]. We note that the lattice $L I_{G}$ is isomorphic to the lattice of all group coarse structure on $G$ (or group balleans in terminology of [9]). Every group coarse structure $\mathcal{E}$ on $G$ has the base $\left\{(x, y): x^{-1} y \in A\right\}$, $A \in \mathcal{I}$ where $\mathcal{I}$ is a group ideal in $\mathcal{P}_{G}$.

For every Abelian group $G$, the lattice $L I_{G}$ is modular, but $L I_{F}$ is not modular for a free non-Abelian group $F$. It would be interesting to find a lattice-theoretical property valid for each $L I_{G}$ and, more generally, for every lattice $\mathcal{L}_{X}$.

4. By analogy with topological groups, a group coarse structure $\mathcal{E}$ on a group $G$ is called maximal $\mathcal{E}$ is a maximal unbounded coarse structure on $G$. These structures were introduced 
and studied in [6], see also [9, Section 10.3]. If a group $G$ admits a maximal group coarse structure $\mathcal{E}$ then the set $\left\{y^{2}: g \in G\right\}$ is bounded in $\mathcal{E}$. It follows that, say, $\mathbb{Z}$ does not admit such a structure. Under $C H$, the maximal group coarse structure on the countable Boolean group was constructed in [6], see also [9, Example 10.3.2]. As to our knowledge, the question whether or not, a maximal group coarse structure can be constructed in ZFC with no additional set-theoretical assumptions remains open.

5 . Let $\Gamma$ be a connected graph with the set of vertices $\mathcal{V}$. We replace each edge $\{u, v\}$ of $\Gamma$ to three edges $\left\{u, x_{\{u, v\}}\right\},\left\{x_{\{u, v\}}, y_{\{u, v\}}\right\},\left\{y_{\{u, v\}}, y\right\}$ and get the subdivision $\Gamma^{\prime}$ of $\Gamma$. We denote by $d$ the pass metric on the set $\mathcal{V}^{\prime}$ of vertices of $\Gamma^{\prime}$. The partition $\left\{B_{d}(v, 1): v \in \mathcal{V}\right\}$ of $\mathcal{V}^{\prime}$ satisfies Proposition 10 so $\mathcal{E}_{\Gamma^{\prime}}$ is $\vee$-decomposable.

We note that $\mathcal{E}_{\Gamma}$ is uniformly locally finite if and only if there exists $m \in \mathbb{N}$ such that $\lambda(v) \leq m$ for each $v \in \mathcal{V}$, where $\lambda(v)$ is the local degree of $v$. By above paragraph, if $\mathcal{E}_{\Gamma}$ is uniformly locally finite then there exist a set $\mathcal{V}^{\prime}, \mathcal{V} \subset \mathcal{V}^{\prime}$ and a uniformly locally finite structure $\mathcal{E}^{\prime}$ on $\mathcal{V}^{\prime}$ such that $\mathcal{E}^{\prime}$ is $\vee$-decomposable, $\mathcal{V}$ is a large subset of $\left(\mathcal{V}^{\prime}, \mathcal{E}^{\prime}\right)$ and $\left.\mathcal{E}^{\prime}\right|_{\mathcal{V}}=\mathcal{E}_{\Gamma}$.

6. Can every metric uniformity on a set $X$ be represented as supremum of two ultrametric uniformities? As to our knowledge, this question was nowhere asked.

We show that, for every set $X$, the metric uniformity $\mathcal{M}$ of $\ell_{\infty}(X)$ is supremum of two ultrametric uniformities. We denote $P=\left\{f \in \ell_{\infty}(X): 0 \leq f<1\right\}, R=\left\{f \in \ell_{\infty}(X):-\frac{1}{2} \leq\right.$ $\left.f<\frac{1}{2}\right\}$, shift $P$ and $R$ by all integer valued functions from $\ell_{\infty}(X)$ and get the partitions $\mathcal{P}$ and $\mathcal{R}$ of $\ell_{\infty}(X)$. For each $n \in \omega$, we denote $\mathcal{P}_{n}=\left\{\left(\frac{1}{2}\right)^{n} P^{\prime}: P^{\prime} \in \mathcal{P}\right\}, \mathcal{R}_{n}=\left\{\left(\frac{1}{2}\right)^{n} R^{\prime}: R^{\prime} \in \mathcal{R}\right\}$. Then $\left\{\mathcal{P}_{n}: n \in \omega\right\}$ and $\left\{\mathcal{R}_{n}: n \in \omega\right\}$ define ultrametric uniformities $\widetilde{\mathcal{P}}$ and $\widetilde{\mathcal{R}}$ such that $\mathcal{M}=\widetilde{\mathcal{P}} \widetilde{\mathcal{R}}$, in particular, $\mathcal{M}=\sup \{\widetilde{\mathcal{P}}, \widetilde{\mathcal{R}}\}$. If we use the families of partitions $\left\{\mathcal{P}_{n}^{\prime}: n \in \omega\right\}$, $\left\{\mathcal{R}_{n}^{\prime}: n \in \omega\right\}$, where $\mathcal{P}_{n}^{\prime}=\left\{2^{n} P^{\prime}: P^{\prime} \in \mathcal{P}\right\}, \mathcal{R}_{n}^{\prime}=\left\{2^{n} R^{\prime}: P^{\prime} \in \mathcal{R}\right\}$, then get $\vee$-decomposition of the coarse structure of $\ell_{\infty}(X)$ in two cellular. Every metric space $(X, d)$ can be realized isometrically as some subspace of $\ell_{\infty}(X)$ but we do not know if decomposability is inherited by subspaces of $\ell_{\infty}(X)$ neither in coarse nor in uniform cases.

\section{REFERENCES}

1. D. Dikranjan, N. Zava, Some categorical aspects of coarse structures and balleans, Topology Appl., 225 (2017), 164-194.

2. A. Dranishnikov, Asymptotic topology, Russian Math. Surveys, 55 (2000), 1085-1129.

3. M. Filali, Ie. Lutsenko, I. Protasov, Boolean group ideal and the ideal structure of $\beta G$, Mat. Stud., 31 (2009), 19-28.

4. P. Harpe, Topics in geometrical group theory, University Chicago Press, 2000.

5. O.V. Petrenko, I.V. Protasov, Balleans and G-spaces, Ukr. Math. Jh., 64 (2012), 344-350.

6. I.V. Protasov, Balleans of bounded geometry and G-spaces, Mat. Stud., 30 (2008), 61-66.

7. I. Protasov, T. Banakh, Ball structures and colorings of groups and graphs, Math. Stud. Monogr. Ser., V.11, VNTL, Lviv, 2003.

8. I.V. Protasov, O.I. Protasova, Sketch of group balleans, Math. Stud., 22 (2004), 10-20.

9. I. Protasov, M. Zarichnyi, General Asymptopogy, Math. Stud. Monogr., V.12, VNTL, Lviv, 2007.

10. O.I. Protasova, Maximal balleans, Appl. Gen. Topology, 7 (2006), 151-163.

11. J. Roe, Lectures on Coarse Geometry, AMS University Lecture Ser.31, Providence, RI, 2003.

Faculty of Computer Science and Cybernetics

Kyiv University Kyiv, Ukraine

i.v.protasov@gmail.com

ksuha@freenet.com.ua 